\title{
NICMOS PERFORMANCE ON THE HUBBLE SPACE TELESCOPE
}

\author{
Rodger I. Thompson ${ }^{\mathrm{a}}$ and Glenn Schneider ${ }^{\mathrm{a}}$ \\ ${ }^{a}$ Steward Observatory, University of Arizona
}

\begin{abstract}
NICMOS, the Near Infrared Camera and Mult-Object Spectrometer was placed in the Hubble Space Telescope in February of 1997. Since then it has been carrying out and extensive program of scientific research. This paper presents the current status and performance of the instrument along with a sample of the observations that have been carried out to this date.
\end{abstract}

Keywords: Infrared, Hubble Space Telescope, Astrophysics

\section{INTRODUCTION}

NICMOS provides imaging, spectroscopic, polarimetric and coronagraphic observations in the near infrared (1.0 - 2.5 microns) with HST. Its primary purpose is diffraction limited high resolution imaging throughout the spectral region and exploitation of the lack of strong telluric OH emission at the HST orbital altitude. Experience with the NICMOS 3 infrared detectors developed for the mission indicated response at wavelengths as short as 0.8 microns, therefore, filters were added to utilize this capability. This provides an overlap with the WFPC and STIS wavebands for linking observations from ultraviolet through the near infrared. Thermal emission from the warm HST mirrors limits observations beyond 2.5 microns so the detectors were tuned to cutoff at that wavelength. This manuscript is an update of the information presented in Ref. 1. The data at the time of that submission was the status in March 1997 whereas this represents the status as of February 1998, approximately 1 year after the launch of NICMOS.

\section{OPTICAL PERFORMANCE}

All three of the NICMOS cameras have demonstrated excellent image during the several thousands of images that exist to date. At this time camera 3 still requires a small ( 21 micron) motion of the HST secondary mirror for optimal performance. Without this adjustment the flux in the central pixel is down by about $25 \%$ of the optimal value. We also have a good characterization of the detector performance parameters which are discussed in section 2.2. Table 1 gives the imaging characteristics of the three cameras. The difference between the $x$ and $y$ pixel scales is due to the tilt of the image plane at the location of the detectors. This is primarily due to the off-axis placement of NICMOS. The difference was not considered significant enough to tilt the detectors in compensation for the field angle.

Table 1. Updated NICMOS camera parameters.

\begin{tabular}{|c|c|c|c|c|c|c|c|c|}
\hline Camera & $\begin{array}{c}\text { X Pixel } \\
\text { arc sec }\end{array}$ & $\begin{array}{c}\text { Y Pixel } \\
\text { arc sec }\end{array}$ & $\begin{array}{c}\text { X FOV } \\
\text { arc sec }\end{array}$ & $\begin{array}{c}\text { Y FOV } \\
\text { arc sec }\end{array}$ & $\begin{array}{c}\text { P.A. V3 } \\
\text { degrees }\end{array}$ & $\begin{array}{c}\text { Dark Cur. } \\
\text { elec/sec }\end{array}$ & $\begin{array}{c}\text { Read Noise } \\
\text { electrons }\end{array}$ & $\begin{array}{c}\text { Gain } \\
\text { e/ADU }\end{array}$ \\
\hline 1 & 0.04323 & 0.04305 & 11.07 & 11.02 & 315.33 & $0.16 \mathrm{e} / \mathrm{s}$ & $22 \mathrm{e}$ & 5.4 \\
\hline 2 & 0.07605 & 0.07537 & 19.47 & 19.29 & 314.52 & $0.15 \mathrm{e} / \mathrm{s}$ & $29 \mathrm{e}$ & 5.4 \\
\hline 3 & 0.20383 & 0.20308 & 52.18 & 51.99 & 314.86 & $0.15 \mathrm{e} / \mathrm{s}$ & $30 \mathrm{e}$ & 6.5 \\
\hline
\end{tabular}

\subsection{IMAGE QUALITY}

The observed image quality in cameras 1 and 2 match well with the expected image quality. Figure 1 compares the encircled energy as a function of radial distance for a point spread function (PSF) computed using the software program TINY TIM (Ref. 2) and the encircled energy measured for a star image in camera 2 . The filter was the broad band filter F160W which has a center wavelength slightly shorter than the camera 2 diffraction limit wavelength of 1.75 microns. We define the diffraction limit as the wavelength where the camera has five pixels between the first zeros of the theoretical PSF for HST. Figure 1. shows that the actual encircled energy function is very close to the theoretical function.. 


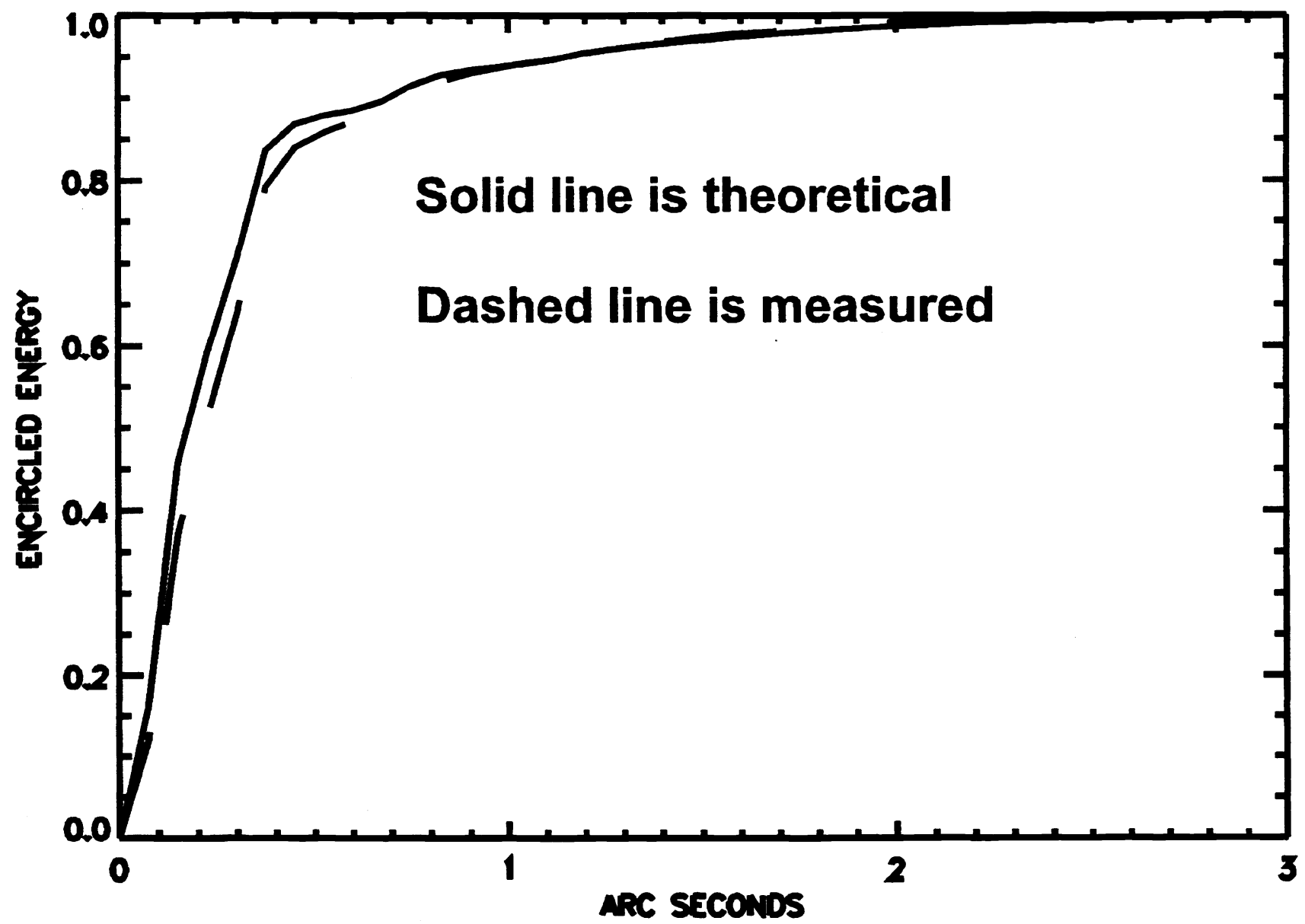

Figure 1. The encircled energy plot for camera 2.

A special issue of the Astrophysical Journal Letters contains several plates and figures that demonstrate the image quality of the NICMOS cameras. Figure 2 is from Ref. 4 , in this figure note the detection of the several faint stars 3 to 4 arc seconds away from a bright source nearly $10^{4}$ times brighter than the faint stars. This clearly shows the low scattering background in NICMOS and the advantages of detectors that do not leave trails of residual image near very bright objects due to charge transfer effects. 


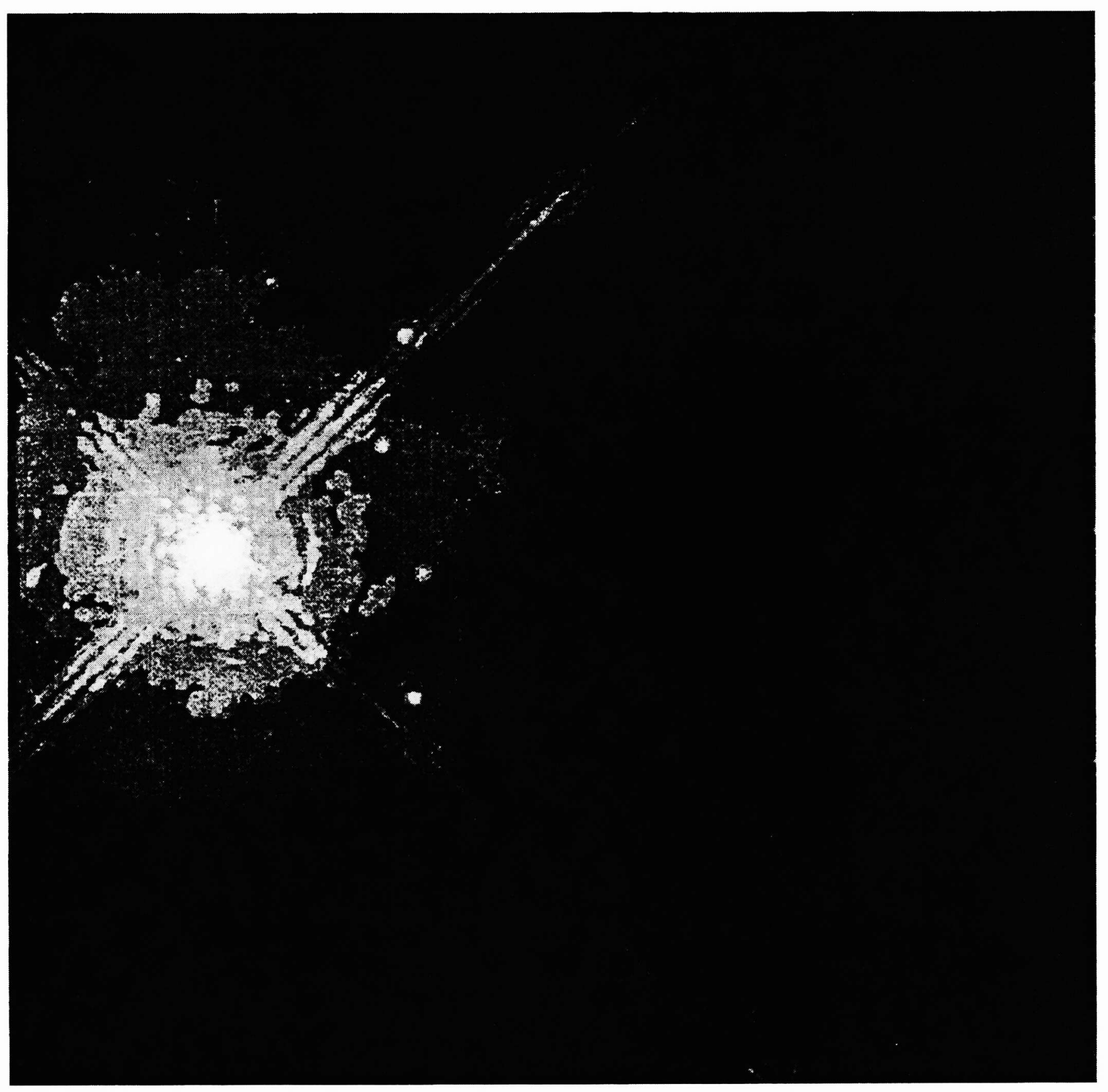

Figure 2. Faint stars around the bright star NGC 2264 IRS

The recent camera 3 campaign demonstrated the excellent image quality of camera 3 when the HST secondary mirror is in the proper position for camera 3 focus. The three week campaign produced several thousand NICMOS camera 3 images of superb quality. If camera 3 does not relax as cryogen is lost to the focus range of the instrument there will probably be another camera 3 campaign in the July time frame. 


\subsection{PHOTOMETRIC PERFORMANCE}

The photometric performance of NICMOS is close to the prelaunch estimates of the NICMOS Instrument Definition Team (IDT). The Exposure Time Calculation tool maintained by the Space Telescope Science Institute (STScI) has been revised to reflect the true on orbit performance of the instrument. Table 1 gives some of the basic photometric parameters for three cameras. These include the dark current, read noise and gain. On orbit measurements show that the background infrared flux levels are less than the levels expected before flight. This means that the cold baffling of the detectors is working well and that there are no unexpected thermal sources in the HST itself. In some regions of the sky the background flux in the 0.2 arc second pixels of camera 3 in the F160W filter is as low as 0.5 electrons per second.

To date none of the basic parameters such as noise, dark current or gain have changed while in orbit. In a period of 1 year of monitoring in orbit there has been no change in the dark current due to the action of cosmic rays on the detector. There also has been no change in the number of electrically bad pixels due to cosmic ray damage.

The total photometric performance is a combination of all of the photometric parameters. SMOV activities included photometric tests in selected filters. The tests produced images of a standard star at three different locations on the array in four to five filters per camera. Table 2 gives the performance levels of the cameras in the measured filters along with the expected sensitivity for a 1000 second integration on a point source. The sensitivity number is expressed as the flux of a point source in Janskys that will give a signal-to-noise ratio of 1 in a full aperture of 6 Airy rings photometric reduction.

Table 2. NICMOS Camera Performance Figures

\begin{tabular}{|c|c|c|c|c|}
\hline Camera & Filter & $\begin{array}{c}\text { Sensitivity } \\
((\mathrm{e} / \mathrm{sec}) / \mathrm{Jy})\end{array}$ & $\begin{array}{c}\text { Background } \\
(\mathrm{e} / \mathrm{sec})\end{array}$ & $\begin{array}{c}\text { S/N = 1 Flux } \\
(\text { Janskys })\end{array}$ \\
\hline 1 & F090M & $3.7210^{5}$ & 0.1 & $1.8310^{-7}$ \\
\hline 1 & F110M & $7.1010^{5}$ & 0.11 & $9.5110^{-8}$ \\
\hline 1 & F145M & $9 . .2510^{5}$ & 0.13 & $7.0010^{-8}$ \\
\hline 1 & F165M & $1.1210^{6}$ & 0.20 & $5.9310^{-8}$ \\
\hline 2 & F110W & $2.3710^{6}$ & 0.30 & $3.6210^{-8}$ \\
\hline 2 & F165M & $1.1610^{6}$ & 0.26 & $7.3910^{-8}$ \\
\hline 2 & F207M & $8.4910^{5}$ & 0.59 & $2.2910^{-7}$ \\
\hline 2 & F222M & $8.9310^{5}$ & 9.0 & $3.9110^{-7}$ \\
\hline 2 & F237M & $1.1210^{6}$ & 39.0 & $6.2610^{-7}$ \\
\hline 3 & F110W & $2.1210^{6}$ & 0.42 & $3.3310^{-8}$ \\
\hline 3 & F160M & $2.1710^{6}$ & & $3.6410^{-8}$ \\
\hline 3 & F166N & $9.7610^{4}$ & & $5.4510^{-7}$ \\
\hline 3 & F222M & $9.0010^{5}$ & & $7.7610^{-7}$ \\
\hline 3 & F240M & $1.4710^{6}$ & & $1.4110^{-6}$ \\
\hline
\end{tabular}

Measured background levels are not given for most filters camera 3 since these are just now being measured from observations taken during the camera 3. The observations to date indicate the new Field Offset Mirror position discussed in Ref. 4 has greatly reduced the thermal background contribution as expected. The background levels now measured in camera 3 are even lower than predicted.

\subsubsection{Noise and Systematic Effects}

During the last three weeks in January 1998 NICMOS performed very deep observations of the Hubble Deep Field previously observed by the Wide Field and Planetary Camera. The observations consisted of 127 observations of 896 seconds in the F160W and F1 10W filters along with additional spectroscopic observations. Preliminary analysis of these observations indicate that the expected signal to noise gain from the addition of images is achieved and their are no systematic effects that limit the achievable signal to noise. We expect that this will be the longest series of observations 
taken with NICMOS so observers should expect that they can attain the expected signal to noise values in their observations.

\subsection{Photometric Features}

At this time there are two epochs of NICMOS data. Data taken after a flight software fix on Aug. 22,1997 and data taken previous to that. Investigators processing NICMOS images should be aware of a feature in the pre Aug. 221997 data which, if not compensated for, could compromise the quality of the final results. This feature is a residual charge present in images taken after NICMOS has been in the "auto-flush" mode. Any time a detector is idle it reverts to this mode, therefore the first reads of any sequence of observations are subject to this effect. The residual charge is on the order of 30 to 40 ADUs ( 200 electrons) per pixel. The second image has about half this signal and subsequent images have little or no residual. In this context an image means a full multi-accum or accum sequence, not the individual reads in a sequence. The proper way to address this feature is to subtract the first dark in a dark sequence from the first image in an image sequence, the second dark from the second image, then the median of the remaining darks in a sequence from the remaining images. If a sequence has spatial dithering NICMOS goes into the auto-flush mode while the telescope is moving. If the move is on the order of an arc second or less the residual is partially restored and usually an average of the first and second darks is the proper dark to subtract from the remaining dithered images. This must be done by the observer as the STScI pipeline does not know the sequence of images and darks. Column 127 (first column is 0 ) is particularly sensitive to this and images that do not properly compensate for the effect can have a cosmetically annoying stripe along the column. This has been termed the "pedestal effect" by STScI. Data taken after Aug. 22, 1997 has this effect greatly reduced due to better handling of the auto-flush mode, but in some images the effect can still be seen at a low level

A second effect is only present in images that have been heavily saturated in the first one or two reads of a multi-accum sequence. The NICMOS arrays are divided into quadrants that are read-out simultaneously. When the readout encounters a heavily saturated area there is a slight change in readout level in all rows. This produces an electrical offset that appears as a stripe in the columns that are saturated and their counterparts in the other quadrants. The figure 2 shows this effect. Most observations will not encounter this effect as it requires a high degree of saturation in the first few reads to be noticeable.

\subsection{Spectroscopy}

Three grisms located in the camera 3 filter wheel provide multi-object spectroscopy at all wavelengths. During the recent camera 3 campaign excellent spectra of standard stars were taken. At this point we are in the process of calibrating this data to provide meaningful methods for reducing the grism data. Since the flux on a pixel is monochromatic, each pixel must have a full characterization of its spectral response. This calibration is underway along with an accurate characterization of the grism spectral transparency function. Background levels in each of the grisms are consistent with the equivalent filters.

\subsection{Polarization}

The NICMOS cameras 1 and 2 each contain a set of 3 Polarcore polarizers on a wide band filter. The polarization angles are set roughly 120 degrees apart with band passes of 0.81 to 1.29 microns and 1.9 to 2.1 microns for camera 1 and 2 respectively. Preflight thermal vacuum testing indicated that the polarizers did not have equal efficiencies and that the position angles between each polarizer differed slightly from the nominal 120 degrees. For each polarizer the efficiency across the field of view is constant and the instrumental polarization for both cameras is $\sim 1 \%$, indicating very excellent optical coatings. Table 3 lists the results. 
Table 3. NICMOS polarization parameters.

\begin{tabular}{|c|c|c|}
\hline Polarizer & Efficiency & Position Angle \\
\hline POL0S & 0.972 & ---- \\
\hline POL120S & 0.477 & 114.88 \\
\hline POL240S & 0.768 & 142.42 \\
\hline POLOL & 0.731 & $-\cdots$ \\
\hline POL120L & 0.629 & 122.58 \\
\hline POL240L & 0.874 & 116.76 \\
\hline
\end{tabular}

The unequal efficiencies and non-ideal position angle offsets require a modification of the simple reduction procedure outlined in the NICMOS manual. This modified procedure was successfully tested on camera 2 observations of the highly polarized Egg Nebula obtained during the Early Release Observations Ref. 4 which reveal a wealth of structure not previously seen. This reference also contains a description of the modified method. In areas of the Egg Nebula where the structure appears fairly uniform, the variation in the polarization implies $1 \sigma \sim 5 \%$. Based on our current understanding of the system, observations using the polarizers in Camera 1 and 2 of NICMOS are capable of providing high spatial resolution polarization information for bright and highly polarized objects with uncertainties of $1 \sigma \sim 5 \%$.

\section{CORONAGRAPHIC OBSERVATIONS}

Camera 2 has a coronagraphic capability comprised of two optical elements. The camera 2 field divider mirror contains a 160 micron diameter hole which covers $93 \%$ of the encircled energy of a stellar PSF at 1.6 micron. The hole lowers both the diffracted energy in the surrounding region and the down stream scattering. The radius of the occulting spot is 4 pixels (or $0.3 \mathrm{arcsec}$ ) at the detector focal plane. The spot appears at an approximate location of $-3.5 \mathrm{larcsec},+5.5$ arcsec from the $(+\mathrm{Y}, \mathrm{X})$ corner in all camera 2 images. The second element is a cold pupil-plane mask at $\sim 100 \mathrm{Kelvin}$ that masks the primary mirror edge, the secondary, hole, the secondary spider and the hold down pads to further reduce the diffracted energy. The primary purpose of this mask, however, is suppression of thermal emission from the telescope structures. The presentation by Dr. Glenn Schneider on the NICMOS coronagraph contains more detailed information on this mode of NICMOS imaging.

\section{LIFETIME AND CAMERA 3 FOCUS}

As of the time of the submission of this manuscript, February 1998, camera 3 is continuing to return toward the range of focus of the NICMOS instrument. The return is due to cryogen loss in the area that has pushed the focus forward when the cryogen warmed from its preflight cold hold values. The rate of return has been variable as should be expected from the complicated dewar geometry. The rate of return is slowing, however, and a complete return to the range of the instrument focus is in doubt. The large number of parameters from metallurgy to stress analysis make this prediction very tentative. The movement of the FOM position on camera 3 has improved the focus by altering the field position along the saggital plane. The current camera 3 focus is excellent for most wide field scientific problems.

The same forward motion of the dewar cold well that produced the focus problem also produced a thermal contact between the nitrogen cold well and the vapor cooled shield surrounding the nitrogen tank. The resultant increase in thermal conductivity has increased the net heat load on the solid nitrogen cryogen. If the thermal contact persists throughout the lifetime of the instrument the current projected lifetime terminates at the end of January 1999. Since the thermal contact initiated at the same time as the camera moved out of focus range, the contact may release when and if the camera returns to focus. Under these conditions the eventual lifetime will depend on the time of the termination of the thermal contact. 


\section{ACKNOWLEDGEMENTS}

The NICMOS team wishes to thank the dedicated personnel at Ball Aerospace, Rockwell International, NASA Goddard Space Flight Center, NASA Headquarters, and the Space Telescope Science Institute who have worked over the last 13 years to make this mission possible. We also greatly thank the crew of STS 82 who installed NICMOS into HST along with STIS and greatly improved the overall performance of the telescope. NICMOS was built under NASA contract NASA 531289 and this work is supported in part by NASA grant NAG 5-3042. This letter is based on observations with the NASA/ESA Hubble Space Telescope, obtained at the Space Telescope Science Institute, which is operated by the Association of Universities for Research in Astronomy under NASA contract NAS5-26555. We also wish to thank Marcia Rieke of the NICMOS team for providing much of the photometric data.

\section{REFERENCES}

1. R.I. Thompson, M. Rieke, G. Schneider, D.C. Hines, M.R. Corbin, Astrophysical Journal Letters, 492, pL95, 1998.

2. H. Krist ASP Conference Series 77, Astronomical Data Analysis Software and Systems IV ASP San Francisco, 1995.

3. R. Sahai, D. C. Hines, J.H. Kastner, D. A. Weintraub, J.T. Trauger, M.J. Rieke, R.I. Thompson, M. Corbin, E. Young, G. Schneider, Astrophysical Journal Letters, 492, p L163, 1998.

4. R.I. Thompson, M.R. Corbin, E. Young, G. Schneider, Astrophysical Journal Letters, 492, L177, 1998. 